\title{
Influence of Electrically Powered Pedal Assistance on User-Induced Cycling Loads and Muscle Activity during Cycling
}

\author{
Sien Dieltiens ${ }^{1, *}$, Carlos Jiménez-Peña ${ }^{2} \mathbb{D}$, Senne Van Loon ${ }^{3} \mathbb{D}$, Jordi $D^{\prime}$ hondt $^{1} \mathbb{D}$, Kurt Claeys ${ }^{4} \mathbb{D}$ and \\ Eric Demeester ${ }^{5}$
}

check for updates

Citation: Dieltiens, S.; Jiménez-Peña, C.; Van Loon, S.; D’hondt, J.; Claeys,

K.; Demeester, E. Influence of

Electrically Powered Pedal Assistance on User-Induced Cycling Loads and Muscle Activity during Cycling. Appl. Sci. 2021, 11, 2032. https://doi.org/ 10.3390/app11052032

Academic Editors: Redha Taiar and Mario Bernardo-Filho

Received: 5 February 2021

Accepted: 16 February 2021

Published: 25 February 2021

Publisher's Note: MDPI stays neutral with regard to jurisdictional claims in published maps and institutional affiliations.

Copyright: (c) 2021 by the authors. Licensee MDPI, Basel, Switzerland. This article is an open access article distributed under the terms and conditions of the Creative Commons Attribution (CC BY) license (https:// creativecommons.org/licenses/by/ $4.0 /)$.
1 Department of Electromechanical Engineering, KU Leuven Technologiecampus Gent, 9000 Ghent, Belgium; jordi.dhondt@kuleuven.be

2 Department of Materials Engineering, KU Leuven Technologiecampus Gent, 9000 Ghent, Belgium; carlos.jimenezpena@kuleuven.be

3 Department of Physics, Universiteit Antwerpen, 2000 Antwerp, Belgium; senne.vanloon@uantwerpen.be

4 Department of Rehabilitation Sciences, KU Leuven Campus Brugge, 8200 Bruges, Belgium; kurt.claeys@kuleuven.be

5 Department of Electromechanical Engineering, KU Leuven Campus Diepenbeek, 3590 Diepenbeek, Belgium; eric.demeester@kuleuven.be

* Correspondence: sien.dieltiens@kuleuven.be

Abstract: Bicycles with electrically powered pedal assistance (PA) show great potential as ecological alternatives for engine-based vehicles. There is plenty of research available about the influence of various bicycle parameters on cycling technique. Though, to the best of the authors' knowledge, there is none about the influence of PA. In this study, a recreational bicycle is equipped with PA and unique instrumentation to measure the user-induced loads on seat, steer and pedals. Joint loading is derived in the sagittal plane from inverse dynamics and muscle activity of the lower limbs is recorded with an electromyography system integrated in cycling pants. An experiment is set up, in which volunteers cycle on an athletics track, with a varying level of PA and a varying seat height. An ANOVA is conducted to determine significant differences due to the level of PA and seat height and to analyze the interaction effect. No interaction effect was found and only differences due to the level of PA were significant. Knowledge about the influence of PA provides insights into (i) electric bicycle design; (ii) the usage of electric bicycle for physically challenged people; (iii) the usage of electric bicycles as a rehabilitation tool.

Keywords: electric bicycle; load cell; kinetics; kinematics; muscle activity; 5 bar linkage; derived kinetics; joint loading; seat loading; steer loading; pedal loading; EMG; user-induced loading; pedaling

\section{Introduction}

Bicycles are ecological alternatives for engine-based vehicles. Though, due to their relatively small action radius and demand of high physical effort, bicycles are not widely adapted for the everyday commute. Modern bicycles with electrically powered pedal assistance (PA) facilitate higher speeds with less effort, enabling physically challenged people to cycle, increasing the potential of bicycles as a means of transportation. Only little is known about the cycling technique on bicycles with PA. Naturalistic studies point out that when traditional cyclists ride a bicycle with PA (i) their average cycling speed increases by $3-5 \mathrm{~km} / \mathrm{h}$; (ii) they require more reactive maneuvers braking; (iii) their decelerations during sharp braking are higher [1].

Unlike walking, cycling is more consistent since the pedals, steer and seat constrains extremity movements. Cycling technique is often described by the bicycle-cyclist interaction loading on the points of contact, comprising the loads induced by the user on the seat, steer and pedals [2,3]. Forces and moments are measured in the three dimensions 
and related to the pedal position within the pedal cycle. User-induced loads are useful to design bicycle frames and components [4] and to derive lower limb joint loading at the knees, hips and ankles by inverse dynamics [5]. Joint loading patterns are utilized to evaluate cycling for rehabilitation [6], to predict overuse joint injuries [7], to analyze muscle function [8] and to optimize cycling performance [9]. There is plenty of research available about the influence of pedaling cadence [10,11], saddle height [12,13], muscle fatigue [14] and workload $[15,16]$ on joint moments. Though, to the best of the authors' knowledge, there is no information available about the influence of pedal assistance.

As cycling considers a smooth action without great impact, acute injuries are less likely to occur. Overuse injuries on the other hand happen frequently [17], with the most common region being the lower extremities (e.g., the knees and the Achilles tendon). The knee extensor is the prime mover to generate propelling power. Though, overdeveloped knee extensor muscles increase the risk of knee injuries due to muscle imbalance [18,19]. Furthermore, muscle activity patterns are indispensable to analyze cycling technique. Research suggests that muscle activity levels of the lower limbs are influenced by parameters as workload level, pedaling cadence and body posture [20-22]. Though again, no information has been found about the influence of PA on muscle activation patterns in cycling.

The influence of PA on the user-induced loads is determined by using a conventional recreation bicycle by measuring the pedal loads, seat loads and steer loads with validated instrumentation [23-25]. Loads are derived to the lower limb joints with a verified method described by some of the authors in a previous investigation [26]. Muscle activation is measured with a system involving surfaces electrodes integrated in cycling pants, as in [27-29]. Knowledge about the influence of PA on the cycling interaction provides insights of advantages and disadvantages of electric cycling for certain target groups.

\section{Materials and Methods}

\subsection{Participants}

The study was approved by the Ethical Committee research of UZ/KU Leuven. Ten recreational cyclists, six women and four men, volunteered to participate. They were informed about the objectives, protocol and the procedures of the study and an informed consent form was signed. The subjects presented the following mean values: age $31 \pm 5$ years, height $171 \pm 7 \mathrm{~cm}$, body mass $64 \pm 11 \mathrm{~kg}$, Body Mass Index (BMI) $22 \pm 3$. They are all in good physical condition, suffer from no injuries and have a healthy BMI. Subject characteristics are presented in Table 1.

Table 1. Characteristics of the subjects.

\begin{tabular}{ccccccccccc}
\hline & Subject & Subject & Subject & Subject & Subject & Subject & Subject & Subject & Subject & Subject \\
& $\mathbf{1}$ & $\mathbf{2}$ & $\mathbf{3}$ & $\mathbf{4}$ & $\mathbf{5}$ & $\mathbf{6}$ & $\mathbf{7}$ & $\mathbf{8}$ & $\mathbf{9}$ & $\mathbf{1 0}$ \\
\hline Gender & $\mathrm{M}$ & $\mathrm{F}$ & $\mathrm{F}$ & $\mathrm{F}$ & $\mathrm{M}$ & $\mathrm{M}$ & $\mathrm{M}$ & $\mathrm{F}$ & $\mathrm{F}$ & $\mathrm{F}$ \\
Age & 28 & 26 & 33 & 32 & 40 & 28 & 31 & 26 & 30 & 37 \\
Weight & 77 & 62 & 66 & 51 & 80 & 77 & 65 & 49 & 60 & 56 \\
Height & 182 & 172 & 171 & 157 & 173 & 171 & 179 & 164 & 175 & 169 \\
BMI & 23 & 21 & 23 & 20 & 27 & 26 & 20 & 18 & 20 & 20 \\
$\begin{array}{c}\text { Dominant } \\
\text { foot }\end{array}$ & $\mathrm{R}$ & $\mathrm{R}$ & $\mathrm{R}$ & $\mathrm{R}$ & $\mathrm{R}$ & $\mathrm{R}$ & $\mathrm{R}$ & $\mathrm{L}$ & $\mathrm{R}$ & $\mathrm{R}$ \\
\hline
\end{tabular}

\subsection{Experimental Design}

The subjects cycle multiple rounds on an instrumented bicycle on an athletics track with a varying seat post height and level of pedal assistance. The instrumented bicycle contains load cells in the seat, steer and pedals to determine the user-induced loads. The lower limb segments are measured to derive the user-induced forces and moments at the lower limb joints. A mobile surface electromyography system (EMG system) integrated in cycling pants is utilized to analyze muscle activity in the lower limbs. 


\subsection{Instrumentation}

There are three points of contact between the cyclist and the bicycle, namely, the pelvis on the saddle, the hands on the steer and the feet on the pedals. The steer stem, seat post and pedals of a city bike for women with PA are replaced by instrumented ones to measure the user-induced loads on all three points of contact. Encoders are inserted in the pedals to determine pedal orientation and in the bottom bracket to relate the loads to the pedal arm angle. The cycling speed is measured with a reed switch and the muscle activity of the lower limbs are recorded with an EMG system integrated in cycling pants. An overview of the instrumentation is presented in Figure 1.

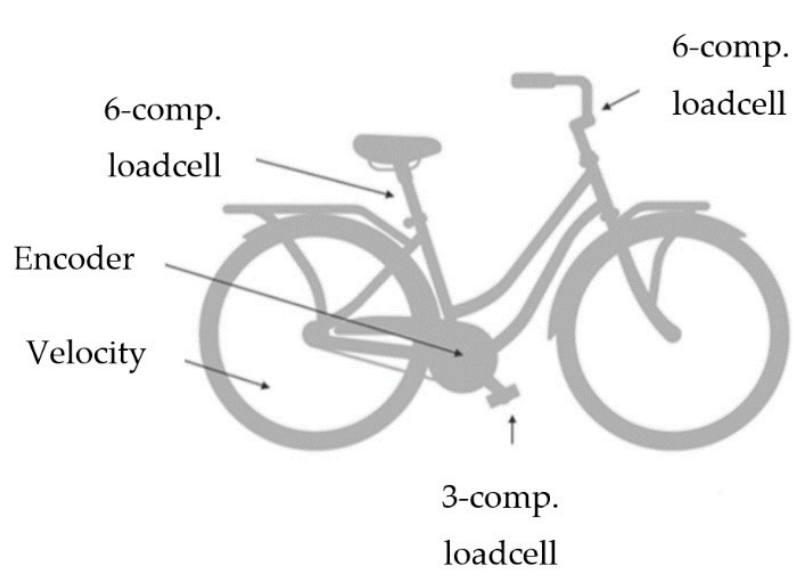

(a)

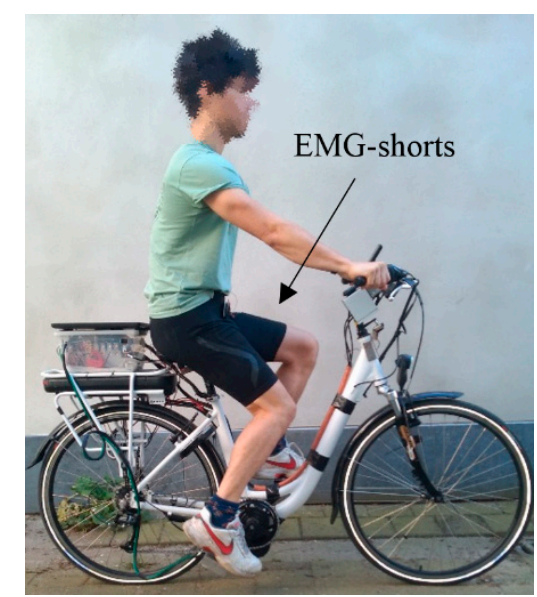

(b)

Figure 1. (a) Schematic overview instrumented bicycle; (b) test subject on instrumented bicycle.

\subsubsection{Bicycle}

All tests are performed under the same circumstances. As there is no control over the delivered power output of the subjects, this parameter is disabled by letting the subjects cycle at a steadily and continuous speed of $20 \mathrm{~km} / \mathrm{h}$ without changing the gears on an electric bicycle, from which the motor power output is based on the cyclist's cadence without taking the pedaling power into account.

A typical city bike for woman is equipped with PA by a conversion kit, namely, the BBS01B of Bafang. The kit contains a pedal assist (PAS) sensor to determine the speed of the spinning bottom bracket, a speed sensor, safety break handles, a battery pack of $36 \mathrm{~V}$ and a middle motor of $250 \mathrm{~W}$ with the following specifications: no-load speed of $85 \mathrm{rpm}$, on-load speed of $81 \mathrm{rpm}$ and a maximal torque of $80 \mathrm{Nm}$. The kit bases the PA on (i) the speed of the bottom bracket, not taking into account the amount of force the cyclist is applying, and (ii) on the level of PA the subject chooses with an interface. There are five degrees of pedal assistance. Depending the cadence of the user, the torque varies from zero until $20 \%$ of the maximal torque output for the first degree, from zero until $40 \%$ for the second degree, from zero until $60 \%$ for the third degree, from zero until $80 \%$ for the fourth degree and from zero until 100\% for the fifth degree. Three PA setups are utilized during testing: no PA (PA 1), PA degree 2 (PA 2) and PA degree 4 (PA 3).

\subsubsection{Instrumented Steer}

The cycling loads exerted by the user's hands are measured with an instrumented steer stem [25]. The stem contains a unique strain gauge based 6-component balance with a maximum standard error of full scale of $0.5 \%$ to measure three dimensional steering forces and moments. 


\subsubsection{Instrumented Seat}

The loads exerted by the user's pelvis are measured with an instrumented seat post [24]. The seat post contains a unique strain gauge based 6-component balance with a maximum standard error of full scale of $1.4 \%$. The pelvis-induced forces and moments are measured in three dimensions.

\subsubsection{Instrumented Pedals}

The pedaling loads exerted by the user's feet are recorded with instrumented pedals [23]. The pedals contain a unique strain gauge based 3-component balance with a maximum standard error of $0.5 \%$ to measure the pedaling forces in three dimensions. The pedal angle is measured with a magnetometer. Two dimensional forces and moments on the lower limb joints are estimated in the sagittal plane utilizing the inverse dynamics model [5].

\subsubsection{Instrumented Bottom Bracket}

The magnitude and direction of the cycling loads is highly dependent of the phase in the pedal cycle. The pedal cycle compromises two phases: the downstroke phase and the upstroke phase. The downstroke phase, when the pedal is pushed down from the highest vertical position (the upper dead center) to the lowest vertical position (the lower dead center), is characterized by the generation of force to propel the bike. The aim of the upstroke phase, when the pedal is lifted from the lowest vertical position to the highest vertical position, is to return the foot to the highest vertical position. Due to the high dependency of the location in the pedal cycle, all cycling loads and muscle activity measurements are related to the position in the pedal cycle. An incremental encoder is installed on the bottom bracket to determine the pedal arm angle [23].

\subsubsection{EMG System}

The muscle activity of the glutes (Gluteus Maximus), hamstrings and quadriceps are measured with a wireless surface EMG system integrated in sport shorts, namely, the Mbody 3 (MYONTEC). Data are collected wireless, internally saved in data acquisition units of the EMG shorts (Mcells) and later uploaded to the computer. The accuracy and validity of the system were proved in earlier studies [27-29].

\subsubsection{Data Acquisition System}

A data acquisition system is integrated in a waterproof box attached to the luggage rack as presented in Figure 2a. The system is directly connected to an 11-inch notebook and controlled by a Matlab script. The bicycle's data acquisition system contains a cDAQ-9178 chassis (NATIONAL INSTRUMENTS), a universal analogue input module NI9219 to read the strain gauge data and a universal analogue input module NI9215 to read the encoder and reed contact data. The magnetometer is connected to an Arduino Micro (ARDUINO). The Mcell of the EMG shorts are adapted for synchronization with the instrumented bicycle, as presented in Figure 2b. A wire is connected from the button of the Mcell to the Arduino Micro. The Arduino continuously sends out a varying signal to the cDAQ and the Mcell to add a unique high-low pattern. After the experiments, all data are shifted in time until they fully match the unique high-low pattern of the Arduino. Furthermore, the Arduino sends a long signal of $5 \mathrm{~s}$ to the Mcell to start and to end a measurement. 


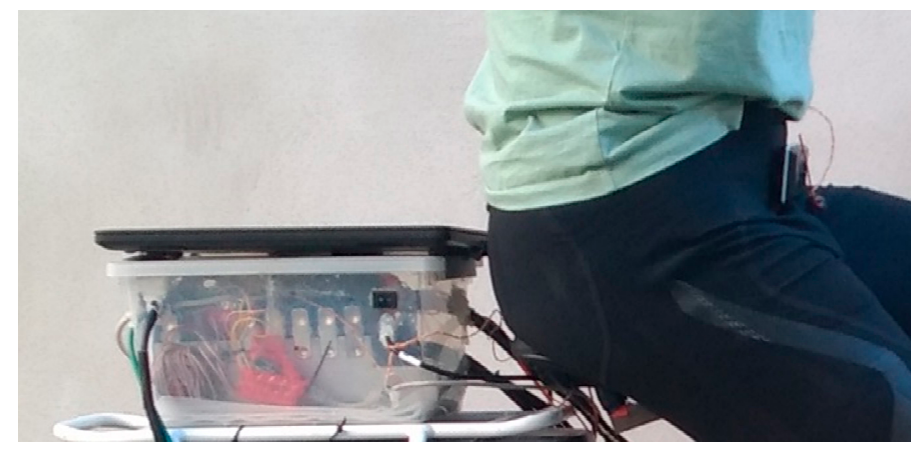

(a)

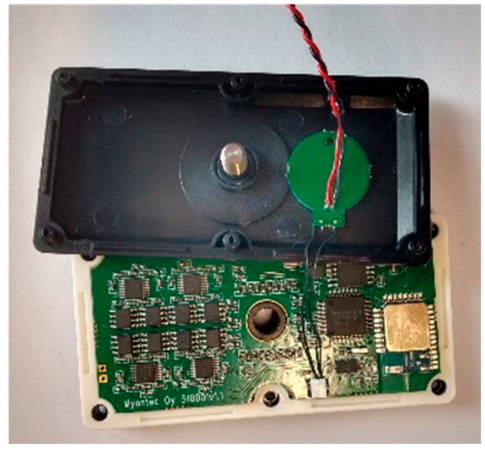

(b)

Figure 2. (a) Data acquisition system in box with notebook on top and wired connected to the Mcell of the EMG shorts; (b) adapted Mcell for synchronization with the compactDaq and Arduino.

\subsection{Protocol}

\subsubsection{Preparation}

For every subject, the weight and body height are measured, the age, pant size and gender are notated, and the BMI is calculated. The lower limb segment lengths of left and right leg are determined according to the methodology of Dieltiens et al. [26], for which (i) the shank length is measured as the distance between the Lateral Femoral Epicondyle and the Lateral Malleolus $\left(D_{\mathrm{S}}\right)$; (ii) the thigh length is measured as the distance between the Greater Trochanter and the Lateral Femoral Epicondyle $\left(D_{\mathrm{t}}\right)$; (iii) the distance between the Medial Malleolus and the Os Naviculare $\left(D_{\mathrm{f}}\right)$ is measured and referred to as the mid foot length.

Each subject is positioned on the bicycle, with the right pedal located in the lower dead center. The feet are placed flat on the pedals with the Os Naviculare in the center. The pedal is orientated horizontally, and the flexion angle of the knee is measured. Based on the method of Holmes and Burke, three seat height positions are determined to create a knee flexion angle of 20 degrees (referred to as SA 1), 25 degrees (SA 2) and 30 degrees (SA 3) [30,31].

Subjects wear EMG shorts with attention for a consistent placement of the electrodes. To maximize the accuracy, the smallest fitting short size is selected, and the electrodes are moistened. There is no tissue allowed between electrodes and skin; therefore, underpants are restricted to strings. The EMG data are normalized by measuring the maximum voluntary contraction (MVC) [32]. MVC normalized data provide an estimation of the neuromuscular effort needed for a given task or exercise. To produce an accurate MVC, all involved segments are stabilized and supported, and an isometric contraction is enforced. A single-joint movement is performed with the segment statically held at middle positions within the range of motion (ROM). Figure 3 addresses the postures in which the MVC is determined for the hamstrings, quadriceps and glutes (Gluteus Maximus).

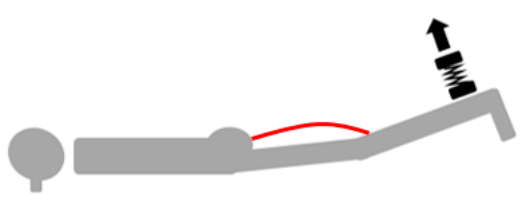

(a)

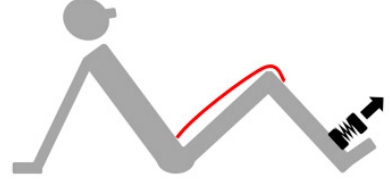

(b)

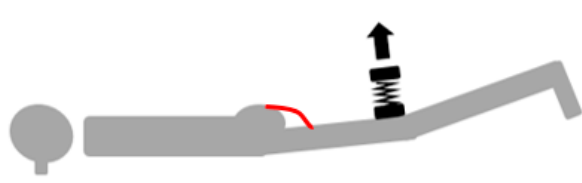

(c)

Figure 3. Maximum voluntary contraction (MVC) experimental design for the (a) hamstrings, (b) quadriceps and (c) glutes.

In advance of each MVC test, the subject warms up for five minutes by stretching and low aerobic exercises. MVC contractions are performed against static resistance. The tests are started slowly, increasing the force, reaching the maximal effort after $3 \mathrm{~s}$. The maximal 
force is held for $3 \mathrm{~s}$ and the subjects relax promptly within $3 \mathrm{~s}$. The test is repeated 4 times with a pausing period of $60 \mathrm{~s}$ in between.

\subsubsection{Tests}

The seat height is set to SA 1 . The subject cycles 3 trials of $5 \mathrm{~min}$. In the first trial, the bicycle is set to PA 1, in the second trial PA 2 and in the third trial PA 3. The test trials are repeated with SA 2 and SA 3. In total, 9 trials are cycled. The subject cycles at a steady and constant speed of $20 \mathrm{~km} / \mathrm{h}$ without changing the gears. The feet are placed flat on the pedals with the Os Naviculare in the center. Both hands are on the steer at all times. The subject only pedals when riding straight. In turns or during maneuvering, the subject stops pedaling and lets the bicycle roll. A researcher cycles next to the subject to give instruction and to correct the subject when necessary.

\subsection{Data Processing}

The data are recorded and analyzed with Matlab. The datasets are synchronized by aligning the Arduino timing with the compact DAQ and the EMG shorts. Erroneous data caused by turning, pedaling backwards, no pedaling, or pedaling unsteadily are removed based on the encoder data. The following requirements are made to save the data: a minimum of five consecutive pedal cycles need to be counted, the deviation between the encoder peaks within the pedal cycle (deviation in cadence) is less than $5 \%$ and the cycling speed is $20 \mathrm{~km} / \mathrm{h}$ with a maximal deviation of $2 \mathrm{~km} / \mathrm{h}$. No individual curves are analyzed. All cycles are averaged to an ensemble average curve (AVC), related to the pedal cycle, for which the original time scale is converted to a percent of a pedal cycle ranging from 0 to $100 \%$.

\subsubsection{Load Cells}

Raw strain load signals are filtered with a third order Butterworth low-pass filter and resolved in $\mathrm{x}, \mathrm{y}$ and $\mathrm{z}$ components utilizing the Least Square Global Regression method [33]. As seen in Figure 4a, initially, the reference frames of the seat, steer and pedals are local. The reference frames of the pedals vary relative to their pedal angle. The local reference frames of the steer and seat are transformed parallel to the bicycle reference frame. Based on the data of the magnetometer and the encoder, the rotating reference frames of the pedals are continuously transformed to the fixed bicycle reference frame, as seen in Figure $4 \mathrm{~b}$. The anterior force follows the $\mathrm{x}$-direction, the normal force follows the z-direction and the lateral force follows the $y$-direction. The axial moment is defined by the $x-y$ plane, the frontal moment by the $y-z$ plane and the sagittal moment by the $x-z$ plane.

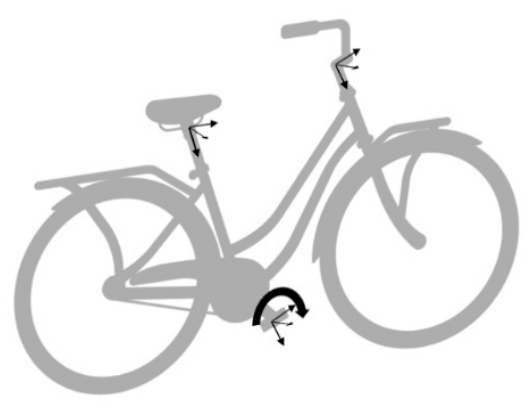

(a)

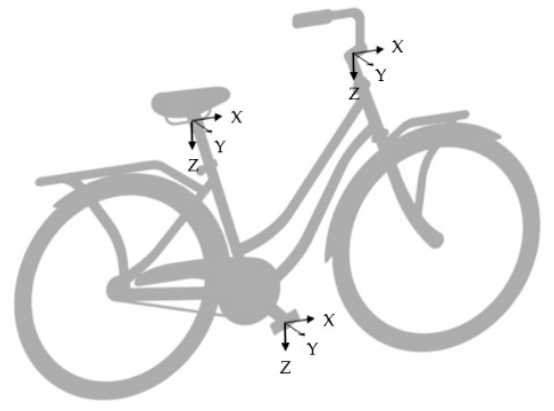

(b)

Figure 4. Transformation of local reference frames (a) to a global reference frame (b).

\subsubsection{Joint Loading}

The lower limb motion of the bicycle-rider system is modelled as a five-bar linkage system, based on the biomechanical model of Hull and Jorge [5], presented in Figure 5. The model singularly considers motion in the sagittal plane, neglecting lateral and axial movement. The bars represent the cyclist's thigh defined as $D_{\mathrm{t}}$, shank defined as $D_{\mathrm{s}}$, 
mid foot defined as $D_{\mathrm{f}}$, bicycle's bottom bracket and the frame as a fixed link. The pivot points are located at the bottom bracket axis (o), pedal axis (p), ankle (a), knee (k) and hip (h). The hip is assumed to be fixed in (h) and the knee joint extends no further than the straight leg position. To specify the linkage configuration, the angular position, velocity and acceleration of the bottom bracket and pedal relative to the pedal spindle are necessary inputs. Based on the linkage configuration and the pedal loading, the anterior and normal forces are calculated in the hips, knees and ankles.

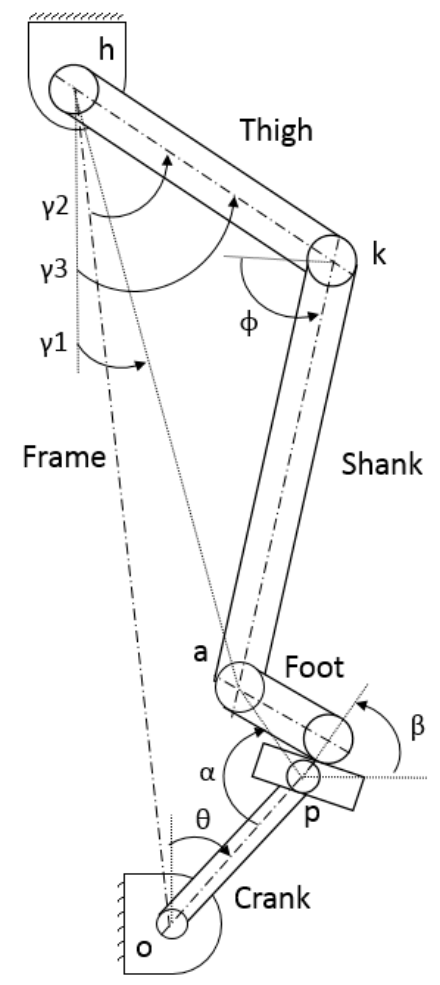

Figure 5. Schematic overview of the bar linkage system from Hull et al.

The kinematics are described in the author's previous work [26]. The kinetics of the hip, knee and ankle are described by Equations (1)-(9).

Ankle:

$$
\begin{gathered}
\boldsymbol{F}_{\boldsymbol{a} x}=m_{f} \boldsymbol{A}_{f x}+\boldsymbol{R}_{n} \sin \alpha+\boldsymbol{R}_{t} \cos \alpha \\
\boldsymbol{F}_{\boldsymbol{a} z}=-m_{f}\left(\boldsymbol{A}_{f z}+g\right)-\boldsymbol{R}_{n} \cos \alpha+\boldsymbol{R}_{t} \sin \alpha \\
\boldsymbol{M}_{\boldsymbol{a}}=+I_{f} \ddot{\beta}-m_{f}\left(\boldsymbol{A}_{f x} C G_{f} \sin \beta+A_{f z} C G_{f} \cos \beta\right)-m_{f} g C G_{f} \cos \beta \\
-L_{f} \cos \beta\left(\boldsymbol{R}_{n} \cos \alpha-\boldsymbol{R}_{\boldsymbol{t}} \sin \alpha\right)-L_{f} \sin \beta\left(\boldsymbol{R}_{t} \cos \alpha+\boldsymbol{R}_{n} \sin \alpha\right)
\end{gathered}
$$

Knee:

$$
\begin{gathered}
\boldsymbol{F}_{k x}=m_{s} \boldsymbol{A}_{\boldsymbol{s} x}+\boldsymbol{F}_{\boldsymbol{a x}} \\
\boldsymbol{F}_{\boldsymbol{k} z}=-m_{s}\left(\boldsymbol{A}_{\boldsymbol{s z}}+g\right)-\boldsymbol{F}_{\boldsymbol{a z}} \\
\boldsymbol{M}_{\boldsymbol{k}}=-M_{a}-I_{s} \ddot{\boldsymbol{\Phi}}-m_{s}\left(\boldsymbol{A}_{s z} C G_{s} \sin \Phi-\boldsymbol{A}_{\boldsymbol{s z}} C G_{s} \cos \Phi\right)-\boldsymbol{F}_{a x} L_{s} \sin \Phi \\
+\boldsymbol{F}_{\boldsymbol{a z}} L_{s} \cos \Phi+m_{s} g C G_{s} \cos \Phi
\end{gathered}
$$

Hip:

$$
\begin{gathered}
\boldsymbol{F}_{h x}=m_{t} \boldsymbol{A}_{t x}+\boldsymbol{F}_{\boldsymbol{k} x} \\
\boldsymbol{F}_{\boldsymbol{h z}}=-m_{t}\left(\boldsymbol{A}_{\boldsymbol{t z}}+g\right)-\boldsymbol{F}_{\boldsymbol{k} z} \\
\boldsymbol{M}_{\boldsymbol{h}}=-M_{k}-I_{t} \ddot{\gamma}-m_{t}\left(\boldsymbol{A}_{\boldsymbol{t} x} C G_{t} \cos \gamma 3+\boldsymbol{A}_{\boldsymbol{t z}} C G_{t} \sin \gamma 3\right)-\boldsymbol{F}_{\boldsymbol{k x}} \cos \gamma 3 \\
-\boldsymbol{F}_{\boldsymbol{k} z} L_{t} \sin \gamma-m_{t} g C G_{t} \cos \gamma 3
\end{gathered}
$$


For which $x$ and $z$ refer to the direction. a, $\mathrm{k}$ and $\mathrm{h}$ refer to the joint (ankle, knee, hip). $f, s$, and $t$ refer to the body parts (foot, shank, thigh). $\boldsymbol{M}_{i}$ is the moment, $\boldsymbol{F}_{i}$ is the force, $\boldsymbol{R}_{\boldsymbol{n}}$ is the normal reaction force, $\boldsymbol{R}_{t}$ is the tangential reaction force, $\boldsymbol{A}_{\boldsymbol{i}}$ is the acceleration, $m_{i}$ is the mass, $L_{i}$ is the length and $C G_{i}$ is the center of gravity.

\subsubsection{Muscle Activity}

Raw EMG signals are exported from muscle monitor into Matlab. The quality of the MVC signals is controlled based on the noise of the baseline signal when the muscles are fully relaxed. The following requirements are made: the amplitude spikes cannot exceed $15 \mu \mathrm{V}$, and the average noise level cannot exceed $5 \mu \mathrm{V}$. The baseline has to stay at the same level before and after contractions. When the muscles are contracted, the EMG signal can reach up to maximally $5000 \mu \mathrm{V}$. The muscle monitor automatically corrects baseline offsets. Additionally, it reflects all negative amplitudes by the baseline to positives ones. The EMG signals are normalized to the MVC, which is calculated as the mean amplitude of the highest signal portion with a duration of $500 \mathrm{~ms}$. It is determined utilizing the moving average technique. Due to the neural interplay and coordination between muscle agonists and antagonists, EMG curves present great standard deviation ranges and can only be interpreted after averaging out multiple cycles to an AVC.

\section{Results}

\subsection{Variables}

Table 2 presents an overview of the measured variables. For the seat and steer, three dimensional forces and moments are recorded. For both pedals, the three-dimensional forces are measured, and the forces and moments of the ankle, knee and hip joints of both legs are derived in the sagittal plane. The muscle activity is determined for the quadriceps, hamstrings and glutes of both legs. All parameters regarding the legs are described for the right leg.

Table 2. List of analyzed variables.

\begin{tabular}{lll}
\hline \multicolumn{1}{c}{ Vight Quadricep Activity (\%max) } & \multicolumn{1}{c}{ Right Hamstring Activity (\%max) } & \multicolumn{1}{c}{ Right Glute Activity (\%max) } \\
\hline Right Ankle Loading: & Right Knee Loading: & Right Hip Loading: \\
Normal force $(\mathrm{N})$ & Normal force $(\mathrm{N})$ & Normal force $(\mathrm{N})$ \\
Anterior force $(\mathrm{N})$ & Anterior force $(\mathrm{N})$ & Anterior force $(\mathrm{N})$ \\
Sagittal moment $(\mathrm{N} \cdot \mathrm{m})$ & Sagittal moment $(\mathrm{N} \cdot \mathrm{m})$ & Sagittal moment $(\mathrm{N} \cdot \mathrm{m})$ \\
Seat Loading: & Steer Loading: & \\
Normal force $(\mathrm{N})$ & Normal force $(\mathrm{N})$ & Right Pedal Loading: \\
Anterior force $(\mathrm{N})$ & Anterior force $(\mathrm{N})$ & Normal force $(\mathrm{N})$ \\
Lateral force $(\mathrm{N})$ & Lateral force $(\mathrm{N})$ & Anterior force $(\mathrm{N})$ \\
Axial moment $(\mathrm{N} \cdot \mathrm{m})$ & Axial moment $(\mathrm{N} \cdot \mathrm{m})$ & Lateral force $(\mathrm{N})$ \\
Frontal moment $(\mathrm{N} \cdot \mathrm{m})$ & Frontal moment $(\mathrm{N} \cdot \mathrm{m})$ & \\
Sagittal moment $(\mathrm{N} \cdot \mathrm{m})$ & Sagittal moment $(\mathrm{N} \cdot \mathrm{m})$ & \\
\hline
\end{tabular}

\subsection{Visual Evaluation AVCs}

AVCs and their standard deviations are determined for all variables in every setup (PA 1-3, SA 1-3). PA 2 and PA 3 are plotted combined with PA 1, similarly SA 2 and SA 3 are plotted together with SA 1 for a visual evaluation. Figure 6 is an example of the AVC for PA 1 combined with PA 2 and PA 3. As seen, the influence by the level of PA is dependent of the phase in the pedal cycle. Four characteristic phases within the pedal cycle are selected to compare the data statistically, namely, the upper dead center $(0 \%)$, the center of the downstroke phase, addressed as the power phase (25\%), the lower dead center $(50 \%)$ and the center of the upstroke phase, addressed as the recovery phase $(75 \%)$. 

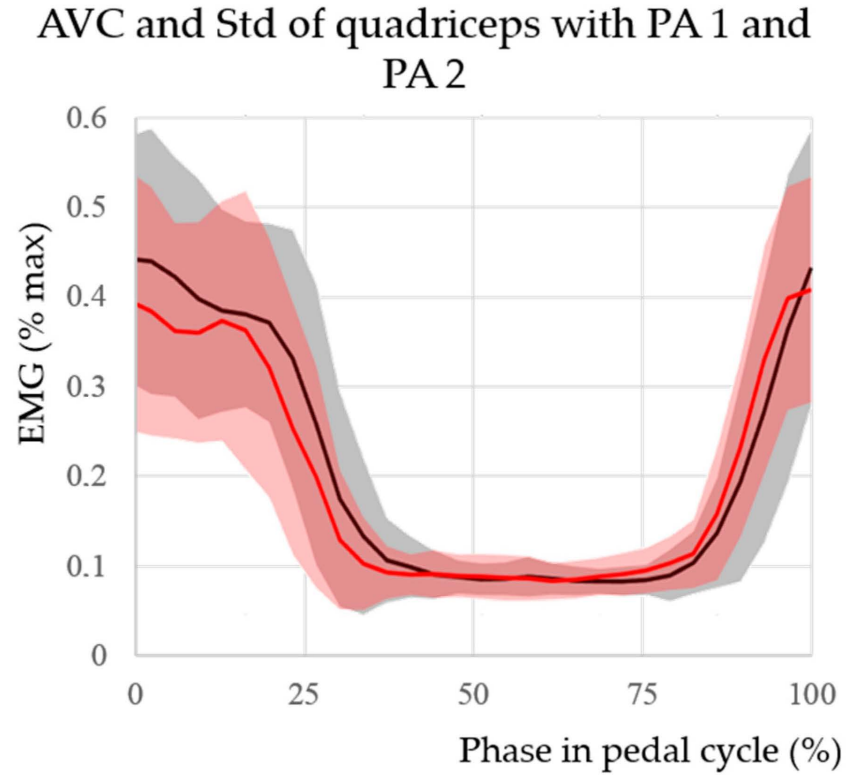

$\square$ PA 1 口PA 2

\section{AVC and Std of quadriceps with PA 1 and PA 3}

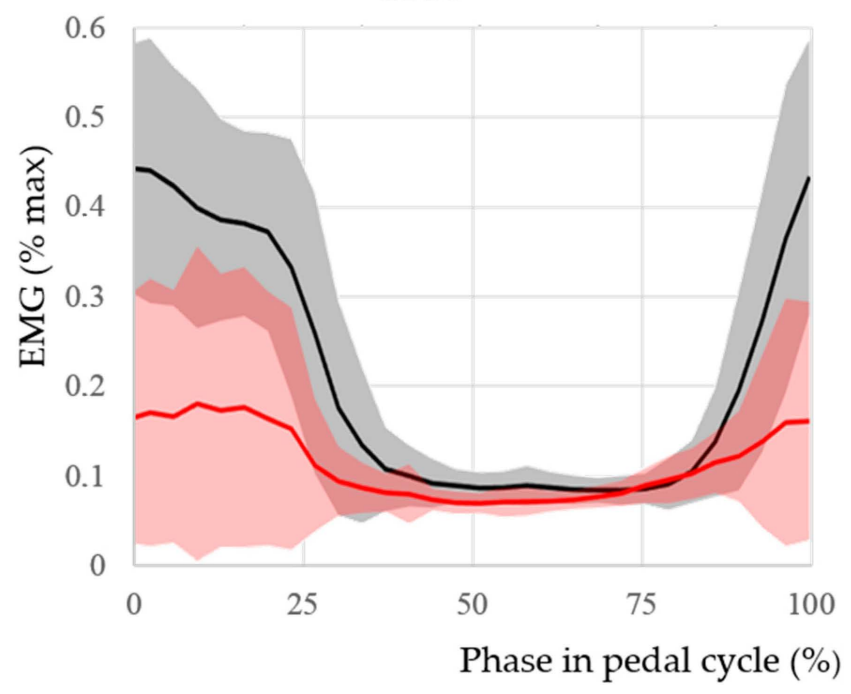

$\square$ PA 1

PA 3

(a)

(b)

Figure 6. Ensemble average curve (AVC) of the quadriceps for pedal assistance (PA) 1 and PA 2 (a); and for PA 1 and PA 3 (b).

\subsection{Statistical Analysis}

A factorial ANOVA is conducted to compare the main effects of (i) different levels of pedal assistance, (ii) different levels of seat heights, and (iii) the interaction effect between both on the variables in the four characteristic phases. The ANOVA reveals that only the level of PA significantly influences the variables; there are no significant influences determined for the seat height. For none of the variables, an interaction effect is found between the level of PA and the seat height. The variables presenting a significant effect (with $p<0.05$ ) are addressed in Figures 7-10. Mean significant differences of the AVCs are presented for the four phases for PA 2 and PA 3, relatively to PA 1. When PA 3 is significant, insignificant differences of PA 2 are also presented, though they have a wider outline. All values are percentages with level 1 as the base line. Error bars are calculated as the percentual ratio between the local standard deviation and the peak to peak amplitude of the whole cycle. Not all visually observed differences are described in the ANOVA due to the high subject-between variance. For this reason, the visually observable differences are also discussed.

\subsubsection{PA in the Upper Dead Center (Phase in Pedal Cycle $=0 \%$ )}

The mean differences of the AVCs of the influence of PA in the upper dead center are addressed by Figure 7. A significant increase in the values due to PA 3 is observed for the steer normal force of $25.5 \%(18.8 \mathrm{~N})$ and for the seat anterior force of $16.3 \%(-17.6 \mathrm{~N})$. A significant decrease in the values due to PA 3 is observed for the hip sagittal moment, hip anterior force, knee sagittal moment, knee anterior force, ankle anterior force, seat lateral force, steer lateral force, steer frontal moment, steer axial moment, the hamstring activity and the quadricep activity of $-158 \%(-55.6 \mathrm{~N} \cdot \mathrm{m}),-83,1 \%(96.6 \mathrm{~N}),-41.3 \%$ $(-21.1 \mathrm{~N} \cdot \mathrm{m}),-84.5 \%(96.3 \mathrm{~N}),-83.8 \%(96.1 \mathrm{~N}),-120.5 \%(-15.7 \mathrm{~N}),-90.1 \%(-9.2 \mathrm{~N})$, of $-67 \%(-0.9 \mathrm{~N} \cdot \mathrm{m}),-69.5 \%(3.8 \mathrm{~N} \cdot \mathrm{m}),-51 \%(-9.7 \% \max )$ and $-64.2 \%(-34.5 \% \max )$, respectively. A significant decrease in the values due to PA 2 is observed for the hip anterior force, knee sagittal moment, knee anterior force, ankle anterior force, steer lateral force, steer frontal moment, hamstring and quadriceps activity of $-45 \%(52.3 \mathrm{~N}),-29.5 \%$ $(-15.1 \mathrm{~N} \cdot \mathrm{m}),-45.8 \%(52.2 \mathrm{~N}),-45.4 \%(52 \mathrm{~N}),-74.9 \%(-7.7 \mathrm{~N}),-44.3 \%(-0.6 \mathrm{~N} \cdot \mathrm{m})$, 
$-27.9 \%(-5.3 \% \max )$ and $-38.3 \%(-20.6 \% \max )$, respectively. Other differences were not significant because of the high standard deviations of the data.

\section{Significant difference by the level of pedal assistance in the upper dead center}

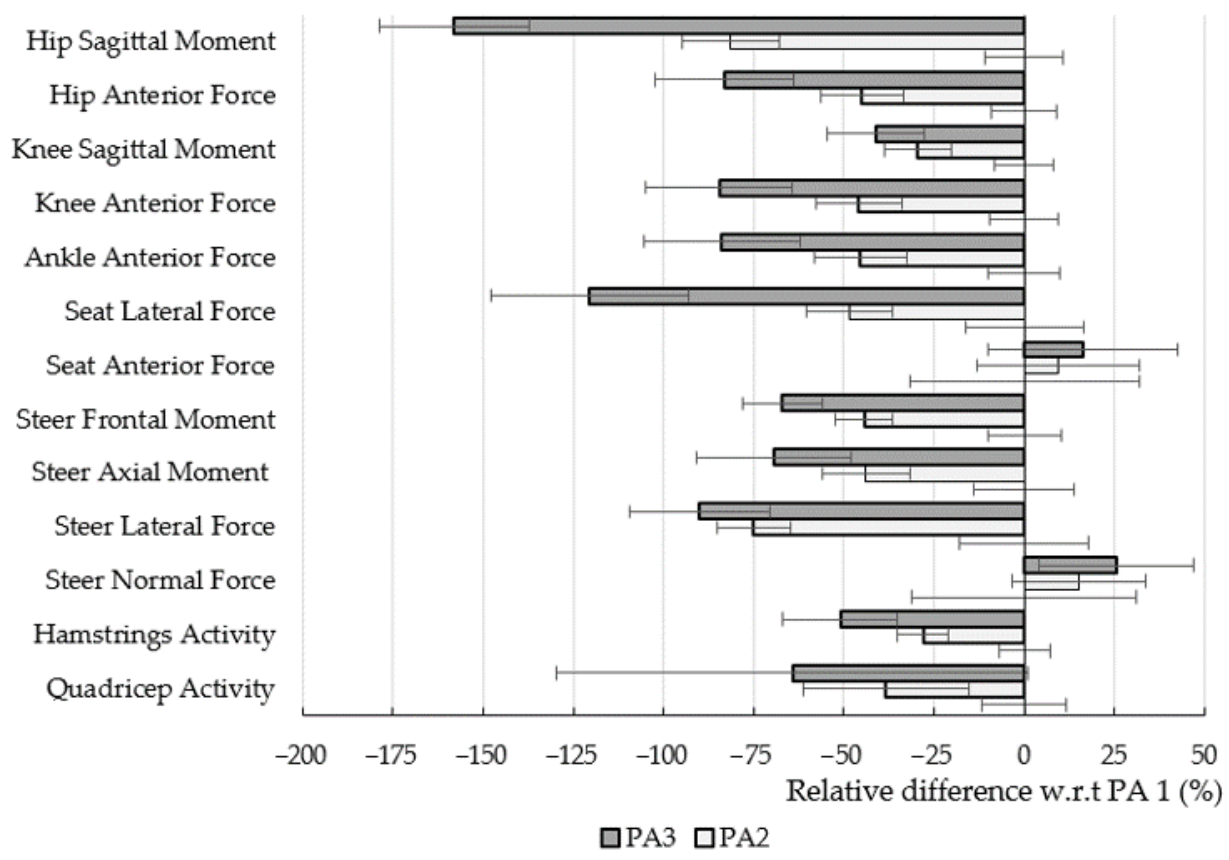

Figure 7. Significant differences based on the level of PA for the upper dead center.

\section{Significant difference by the level of pedal assistance in the power phase}

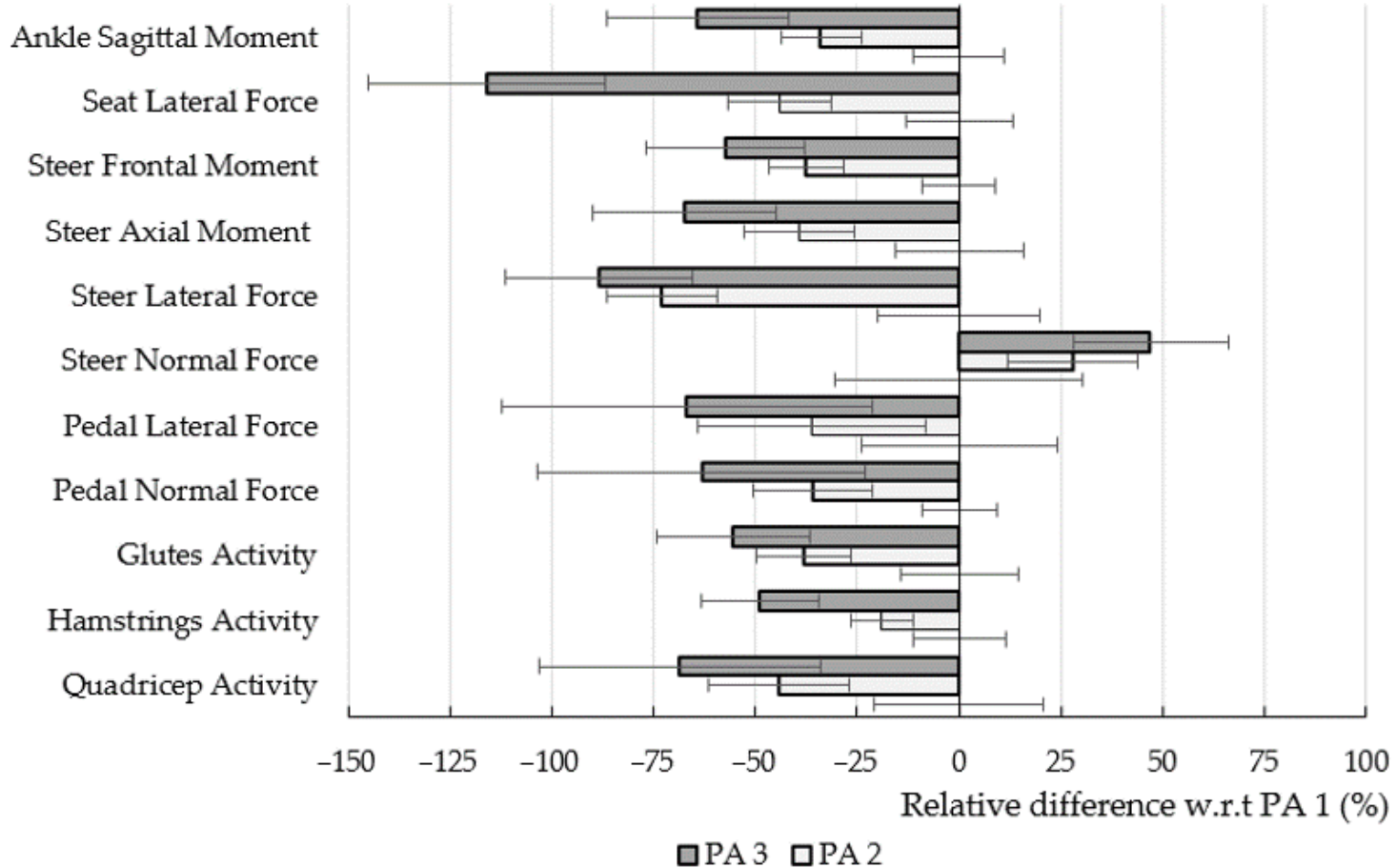

Figure 8. Significant differences based on the level of PA for the power phase. 


\section{Significant difference by the level of pedal assistance in the lower dead center}

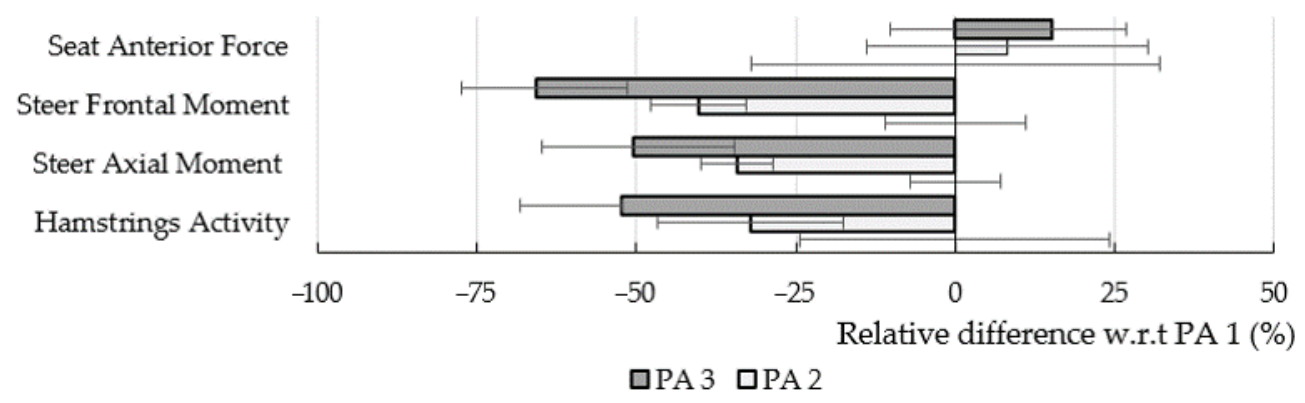

Figure 9. Significant differences based on the level of PA for the lower dead center.

\section{Significant difference by the level of pedal assistance in the recovery phase}

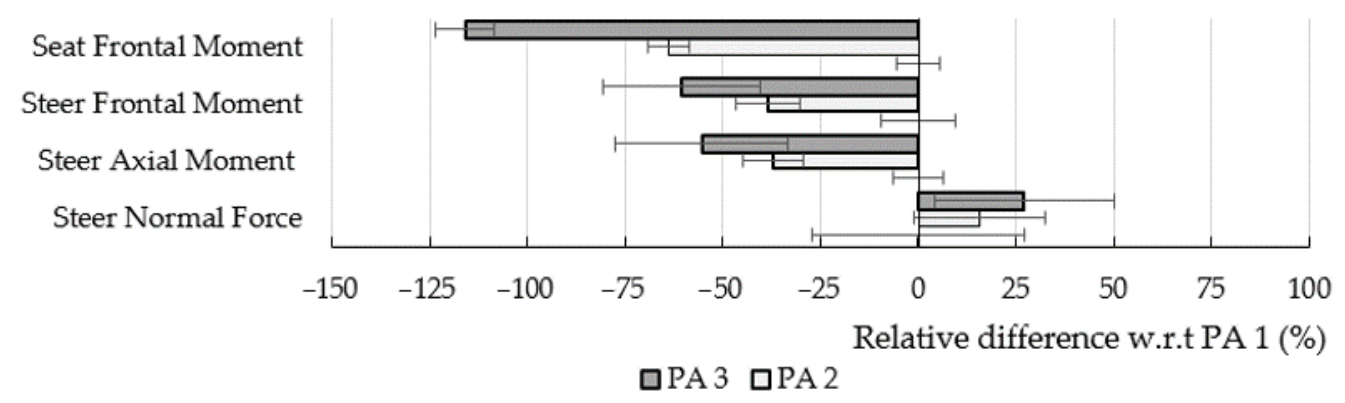

Figure 10. Significant differences based on the level of PA for the recovery phase.

\subsection{2. $\mathrm{PA}$ in the Power Phase (Phase in Pedal Cycle $=25 \%$ )}

The mean differences of the AVCs of the influence of PA in the power phase are addressed by Figure 8. A significant increase for the values due to PA 3 is observed for the steer normal force of $46.9 \%(31.6 \mathrm{~N})$. A significant decrease due to PA 3 is observed for the seat lateral force, ankle sagittal moment, steer lateral force, steer frontal moment, steeraxial moment, the pedal lateral force, the pedal normal force, the glute activity, the hamstring activity and the quadricep activity of $-116 \%(-20.5 \mathrm{~N}),-64.3 \%(-3.6 \mathrm{~N} \cdot \mathrm{m}),-88.4 \%$ $(-9.6 \mathrm{~N}),-57.4 \%(-1 \mathrm{~N} \cdot \mathrm{m}),-67.4 \%(4.2 \mathrm{~N} \cdot \mathrm{m}),-66.8 \%(-8.5 \mathrm{~N}),-63.2 \%(-118.8 \mathrm{~N})$, $-55.5 \%(-12.1 \% \max ),-48.9 \%(-12.8 \% \max )$ and $-68.5 \%(-31.9 \% \max )$, respectively. A significant increase in the values due to PA 2 is observed for the steer normal force of $28.1 \%$ $(18.9 \mathrm{~N})$. Significant decreases due to PA 2 are observed for the steer lateral force, steer frontal moment, ankle sagittal moment, the pedal normal force, the glute activity and the quadriceps activity of $-72.9 \%(-7.9 \mathrm{~N}),-37.7 \%(-0.7 \mathrm{~N} \cdot \mathrm{m}),-34 \%(-1.9 \mathrm{~N} \cdot \mathrm{m}),-35.8 \%$ $(-67.3 \mathrm{~N}),-38.3 \%(-8.3 \% \max )$ and $-44.3 \%(-20.6 \% \max )$, respectively.

\subsubsection{PA in the Lower Dead Center (Phase in Pedal Cycle $=50 \%$ )}

The mean differences of the AVCs of the influence of PA in the lower dead center are addressed by Figure 9. A significant increase in the values due to PA 3 is observed for the seat anterior force of $15 \%(-16.2 \mathrm{~N})$. A significant decrease in the values due to PA 3 is observed for the steer frontal moment, steer axial moment and the hamstring activity of $-65.8 \%(0.7 \mathrm{~N} \cdot \mathrm{m}),-50.6 \%(-3.6 \mathrm{~N} \cdot \mathrm{m})$ and $-52.2 \%(-18.9 \% \max )$, respectively. A significant decrease in the values due to PA 2 is observed for the steer frontal moment, steer axial moment and the hamstring activity of $-40.2 \%(0.4 \mathrm{~N} \cdot \mathrm{m}),-34.2 \%(-2.4 \mathrm{~N} \cdot \mathrm{m})$ and $-32.2 \%(-11.7 \%$ max $)$, respectively. 


\subsubsection{PA in the Recovery Phase (Phase in Pedal Cycle $=75 \%$ )}

The mean differences of the AVCs of the influence of PA in the recovery phase are addressed by Figure 10. A significant increase in the values due to PA 3 is observed for the steer normal force of $27.1 \%(21.6 \mathrm{~N})$. A significant decrease in the values due to PA 3 is observed for the seat frontal moment, steer frontal moment and steer axial moment of $-116 \%(0.5 \mathrm{~N} \cdot \mathrm{m}),-60.5 \%(1 \mathrm{~N} \cdot \mathrm{m})$ and $-55.4 \%(-4.8 \mathrm{~N} \cdot \mathrm{m})$, respectively. A significant increase in the values due to PA 2 is observed for the steer normal force of $15.6 \%(12.4 \mathrm{~N})$. A significant decrease in the values due to PA 2 is observed for the seat frontal moment and steer axial moment of $-63.7 \%(0.3 \mathrm{~N} \cdot \mathrm{m})$ and $-37.1 \%(-3.2 \mathrm{~N} \cdot \mathrm{m})$, respectively.

\section{Discussion}

Changing PA when cycling an e-bike does not only influence the loading exerted by the upper leg muscles but also steer and seat loading. In general, the level of PA does not change the shape of an entire pattern. Significant differences are found, though only in certain phases of the pedal cycle. As most power is exerted in the downstroke phase (from 0 degrees to 150 degrees), most significant differences are found in the upper dead center and power phase. For all analyzed variables, the significant differences escalate in magnitude in function of the amount of pedal assistance. Furthermore, the variation increases in function of the amount of pedal assistance. This is in line with the literature as PA reduces the effort and an increased effort improves the symmetry of pedaling kinetics [34].

The phase in the pedal cycle is addressed from the perspective of the right pedal. The upper dead center and power phase of the right pedal are, respectively, the lower dead center and recovery phase of the left pedal. For this reason, seat and steer AVCs are expected to be symmetric between the two halves of the pedal cycle. Significant differences that are present in the upper dead center or power phase should also be present in, respectively, the lower dead center or the recovery phase. Though, as seen in the Section 3, this is not the case. Only the steer axial moment presents significant differences in opposing phases. When analyzing the AVCs, variation is significantly higher for the upper dead center and power phase of the left pedal, which hinders the significance of differences due to the level of pedal assistance. In total, $90 \%$ of the tested subjects are right-legged, utilizing their right leg as the leader and the left as the follower. Research states that increased pedaling effort demands a more stable technique and results in a reduced variability [35]. Therefore, differences due to PA should be visible in both opposing phases, though will not always be significant. Furthermore, multiple subjects are compared to each other, which increases the variation as each individual has his own cycling technique. Visual differences can be clinically relevant, even though they are not significantly relevant, and will be discussed in this section.

Lateral loading originates from the pedaling movement and is predominantly generated in the downstroke phase. When generating the push on the pedals, the most comfortable power exertion direction is enforced, resulting in a small lateral component. PA reduces the power exertion during the push and, therefore, also the lateral component. In the power phase, a significant decrease in the lateral pedal force is observed due to PA (maximally $-66.8 \%$ ). Lateral seat loading patterns are induced by the pelvis movement. Towards the downstroke phase, the pelvis rotates internally and laterally, causing the hip to translate anteriorly and inferiorly. The movement is presented by the right leg in the first half of the pedal cycle and by the left leg in the second half of the pedal cycle, resulting in locally increasing lateral seat forces. PA significantly decreases the lateral seat forces maximally in the upper dead center (maximally $-120.5 \%$ ) followed by the power phase (maximally $-116 \%$ ). The decrease in the left leg is observable in the visual evaluation, though not significant due to high variation. Lateral steer-loading patterns are induced by trunk motion in the axial plane during pedaling. The trunk rotates medially towards the downstroke phase, generating a pull on the allied handle and a push on the opposing one, resulting in locally increasing lateral steer forces and axial moments. Increasing the level 
of PA, decreases all steer axial moments significantly (maximally $-69.5 \%$ for the upper dead center, maximally $-67.4 \%$ for the power phase, maximally $-50.6 \%$ for the lower dead center and maximally $-55.4 \%$ for the recovery phase), the difference is the largest in the first half of the pedal cycle as the right leg is dominant. The lateral force presents a significant decrease maximally in the upper dead center (maximally $-90.1 \%$ ) followed by the power phase (maximally $-88.3 \%$ ). Decreases in the second half of the pedal cycle are visually observable though not significant due to high variation. The findings suggest that lateral load components reduce in function of the level of pedal assistance, resulting in a reduced pelvis movement and axial trunk movement. Costes et al. state that the accelerations of the trunk center of mass, hips and shoulders increases when cycling at higher workloads [36]. As PA decreases the workload, the results are in line.

The rider's weight is distributed over all points of contact between the bicycle and cyclist. Increasing the level of PA decreases the normal pedal force significantly in the power phase (maximally $-63.3 \%$ ) and insignificantly though observable in the recovery phase. The variation in the recovery phase is notably higher as a lower workload results in higher variation [35]. The normal steer forces are significantly increased in the upper dead center (maximally $25.5 \%$ ), power phase (maximally $46.9 \%$ ), recovery phase (maximally $27.1 \%$ ) and a small insignificant increase is observed in the lower dead center. Decreases in seat loading are visually observable (maximally $-10 \%$ over the whole cycle) though not significant due to high variation (maximally 40\%). An ANOVA was performed with load values adjusted to the weight of the rider to analyze if the difference in body weight is the reason of the high variation. The variation is uninfluenced by the rider's weight, nor by inter-individual differences as gender, age, experience or flexibility level. All subjects present a very high within-subject variation, uninfluenced by the level of pedal assistance, implying they tend to hop around a central value. The findings suggest that the weight distribution over all points of contact between bicycle and cyclist is influenced by the level of pedal assistance. When the reaction forces on the pedals decrease due to an enlarged level of pedal assistance, the body weight is more supported by seat and steer. The same findings are presented by Stone and Hull for high workload levels [2].

Primary movements during pedaling are hip and knee extension in the downstroke phase (0-180 degrees) along with hip flexion in the backstroke phase (180-360 degrees). Knee flexion is less pronounced as it is mostly generated by the knee extension of the leg on the opposing pedal. The glutes extend the hip and are generally active from the upper dead center until the power phase [37]. Due to PA, an insignificant-though clearly visible-decrease in glutes activity is observed in the upper dead center and a significant decrease in the power phase (maximally $-55.5 \%$ ). The quadriceps extend the knee and are generally active from the upper dead center until the power phase [38]. Due to PA, a significant decrease in quadriceps activity is observed in the upper dead center (maximally $-64.2 \%$ ) and in the power phase (maximally $-44.3 \%$ ). The hamstrings are the knee flexors and hip extensors and are active through the whole pedal cycle as they also support the knee extensors to regulate the net joint moments. The literature presents varying results on the activation of the hamstrings. Ercison et al. presented activation between 150 degrees and 270 degrees [20], while Dorel et al. presented activation between 60 degrees and 240 degrees [37]. In this research, the hamstrings are singularly inactive in the recovery phase. An insignificant, though visually noticeable, decrease is observed in the lower dead center and a significant decrease is observed in the upper dead center (maximally 51\%) and the power phase (maximally $-48.9 \%$ ). In the phases where muscles are most active, a decreased activity level in function of the amount of PA is observed. In the phases where muscles are less active, they present no observable differences.

Significant decreases in the joint forces are found in the upper dead center. The ankle, knee and hip anterior forces are significantly decreased to a similar extent (maximally $83.3 \%,-84.5 \%,-83 \%$, respectively). The ankle plantar flexion moment is significantly decreased in the power phase, visually decreased in the lower dead center and visually increased in the upper dead center. Less moment is transferred to the ankle when the foot 
is actively pushing downwards, and more moment is transferred to the ankle when the foot is passively pushed upwards. Accordingly, the knee and hip flexion moments in the upper dead center are significantly decreased (maximally $-41.3 \%$ and $-158 \%$, respectively), and the knee and hip extension moments in the lower dead center are visually increased. In general, due to PA, the significance of the different phases within the pedal cycle is reduced. The motor generates a constant propulsion moment to the pedals, reducing the effort needed to (i) cross the dead centers and (ii) lift the foot in the upstroke phase. This could result in a more limited pedal movement for which the cyclist does not distribute his/her effort over the whole pedal cycle but focusses on the most comfortable phase of the pedal cycle, namely, the extension in the downstroke phase of the pedal cycle.

This concept presents itself furthermore in the anterior forces. Due to PA, the posterior seat forces are significantly increased in the upper dead center (maximally $16.3 \%$ ) and lower dead center (maximally 15.1\%) and visually in the other phases. The posterior steer forces are visually decreased throughout the whole pedal cycle. The findings suggest that the cyclist pulls less on the steer and has a more bent forward posture, which is typically for the lower limb extension movement.

Some of the findings present large percentage differences, though are mechanically and clinically not relevant as the actual values are small. This is the case for the steer axial moment (maximal difference of $4.8 \mathrm{~N} \cdot \mathrm{m}$ ) steer frontal moment (maximal difference of $4.8 \mathrm{~N} \cdot \mathrm{m}$ ), seat frontal moment (maximal difference of $4.7 \mathrm{~N} \cdot \mathrm{m}$ ) and the sagittal ankle moment (maximal difference of $3.6 \mathrm{~N} \cdot \mathrm{m}$ ). The normal and anterior forces present large actual differences which are interesting for mechanical analysis such as electric bicycle frame design. The differences in the normal and lateral forces are clinically interesting as these variables are related to overuse injuries as wrist, bottom, hip and knee [38-40]. PA influences joint loading possibly in a way that the cyclist applies most effort on the most comfortable phase of the pedal cycle and pedal forces and muscle activity are reduced. Therefore, an electric bicycle could be a valuable rehabilitation tool for hip and knee patients and a transportation means for the physically challenged. The normal and posterior forces on the steer and seat increase in function of the level of PA which could imply that electric bicycles are less favorable for people who suffer from wrist, bottom or lower back pain.

\section{Conclusions}

The results of this study revealed that different levels of SA presented no significant influences on the cycling interaction, while different levels of PA presented multiple. No interaction effect was found between both parameters. Most of the significant differences due to PA were found in the upper dead center and power phase, where most pedaling power was exerted. The variation is higher in the lower dead center and recovery phase. A higher amount of PA increases the magnitude of significant differences and the overall variation. Lateral loading of the steer, seat and pedals is reduced in function of the level of PA, suggesting a reduced pelvis and axial trunk movement. The normal forces on the pedals are decreased in function of the level of PA and the normal seat and steer forces are increased. The reaction forces on the pedals are smaller and the body weight is more supported by seat and steer. In the phases that the muscles are most active, the muscles present a decreased activity level in function of the amount of PA. Joint loading also presents local increases as local decreases in function of the amount of pedal assistance. The anterior forces suggest a more bent forward posture with less pulling on the steer. The idea is thus proposed that PA limits the flexion extension action to increased extension action. Results suggest that electric PA could be a valuable rehabilitation tool for hip and knee patients.

Author Contributions: Conceptualization, S.D.; Data curation, C.J.-P. and S.V.L.; Formal analysis, S.D. and C.J.-P.; Funding acquisition, K.C.; Investigation, S.D.; Methodology, S.D., C.J.-P. and K.C.; Project administration, S.D. and K.C.; Resources, E.D.; Software, C.J.-P., S.V.L. and J.D.; Supervision, K.C. and E.D.; Validation, S.D.; Visualization, S.D.; Writing—original draft, S.D.; Writing—review \& 
editing, C.J.-P., S.V.L., J.D., K.C. and E.D. All authors have read and agreed to the published version of the manuscript.

Funding: This research received no external funding.

Institutional Review Board Statement: The study was conducted according to the guidelines of the Declaration of Helsinki and approved by the Ethical Committee research of UZ/KU Leuven. (protocol code S63553, date of acceptance: 16/03/2020).

Informed Consent Statement: Informed consent was obtained from all subjects involved in the study.

Conflicts of Interest: The authors declare no conflict of interest.

\section{References}

1. Huertas-Leyva, P.; Dozza, M.; Baldanzini, N. Investigating cycling kinematics and braking maneuvers in the real world: E-bikes make cyclists move faster, brake harder, and experience new conflicts. Transp. Res. 2018, 54, 211-222. [CrossRef]

2. Stone, C.; Hull, M.L. The effect of rider weight on rider-induced loads during common cycling situations. J. Biomech. 1995, 4, 365-375. [CrossRef]

3. Hull, M.L.; Davis, R.R. Measurement of pedal loading in bicycling: I. Instrumentation. J. Biomech. 1981, 14, 843-856. [CrossRef]

4. Hull, M.L.; Bolourchi, F. Contributions of rider induced loads to bicycle frame stresses. J. Strain Anal. 1988, 23, 105-114. [CrossRef]

5. Hull, M.; Jorge, M. A method for biomechanical analysis of bicycle pedadalling. J. Biomech. 1985, 18, 631-644. [CrossRef]

6. Ericson, M.O.; Nisell, R.; Ekholm, J. Varus and valgus loads on the knee jointduring ergometer cycling. Scand. J. Sports Sci. 1984, $6,39-45$.

7. Ruby, P.; Hull, M.L.; Jenkins, D.; Kirby, K. Effect of anatomy on knee joint loads during seated cycling. J. Biomech. 1992, 25, 1195-1207. [CrossRef]

8. Gregor, R.J.; Cavanaegh, P.R.; LaFortune, M. Knee flexor moments during propulsion in cycling: A creative solution to Lombard's paradox. J. Biomech. 1985, 18, 307-316. [CrossRef]

9. Gonzalez, H.; Hull, M.L. Multivariable optimization of cycling biomechanics. J. Biomech. 1989, 22, 1151-1161. [CrossRef]

10. Neptune, R.R.; Hull, M.L. A theoretical analysis of preferred rate selection in endurance cycling. J. Biomech. 1999, 32, 409-415. [CrossRef]

11. Marsh, A.P.; Martin, P.E.; Sanderson, D.J. Is a joint moment-based cost function associated with preferred cycling cadence? J. Biomech. 2000, 33, 173-180. [CrossRef]

12. Ericson, M.; Bratt, A.; Nisell, R.; Nemeth, G.; Ekholm, J. Load moments about the hip and knee joints during ergometer cycling. Scand. J. Rehabil. Med. 1986, 18, 165-172.

13. Horscroft, R.; Davidson, C.J.; McDaniel, J.; Wagner, B.M.; Martin, J. Effects of saddle height on joint power distribution. Med. Sci. Sport Exerc. 2003, 35, S16. [CrossRef]

14. Mornieux, G.; Guenette, J.A.G.; Sheel, A.W.; Sanderson, D.J. Influence of cadence, power output and hypoxia on the joint moment distribution during cycling. Eur. J. Appl. Physiol. 2007, 102, 11-18. [CrossRef] [PubMed]

15. Caldwell, G.E.; Hagberg, J.M.; McCole, S.D.; Li, L. Lower extremity joint moment during uphill cycling. J. Appl. Biomech. 1999, 15, 166-181. [CrossRef]

16. Ericsson, M. Mechanical muscular power output and work during ergometer cycling at different work loads and speeds. Eur. J. Appl. Physiol. 1988, 57, 382-387. [CrossRef]

17. Dettori, N.; Norvel, D. The effects of exercise on patellar tracking in lateral petellar compression syndrome. Sports Med. 2006, 36, 7-18. [CrossRef] [PubMed]

18. Mariani, P.P.; Caruso, I. An electromyographic investigation of subluxation of the patella. J. Bone Jt. Surg. 1979, 61, 169-171. [CrossRef] [PubMed]

19. Souza, D.R.; Gross, M.T. Comparison of vastus medialis obliquus vastus lateralis muscle integrated electromyographic ratios between healthy subjects and patients with patellofemoral pain. Phys. Ther. 1991, 71, 310-316. [CrossRef]

20. Ericson, M.O.; Nissel, R.; Arborelius, U.P.; Ekhorn, J. Muscular activity during ergometer cycling. Scand. J. Rehabil. Med. 1985, 17, 53-61. [PubMed]

21. Baum, B.S.; Li, L. Lower extremity muscle activities during cycling are influenced by load and frequency. J. Electromyogr. Kinesiol. 2003, 2, 181-190. [CrossRef]

22. Laplaud, D.; Hug, F.; Grelot, L. Reproducibility of eight lower limb muscles activity level in the course of an incremental pedaling exercise. J. Electromyogr. Kinesiol. 2006, 15, 158-166. [CrossRef]

23. Dieltiens, S.; D'hondt, J.; Juwet, M.; Versteyhe, M. Development of A Low-Cost Measurement System to Determine 3-Dimensional Pedal Loads During in-Situ Cycling. Transp. Probl. 2018, 14, 151-160. [CrossRef]

24. Dieltiens, S.; D’hondt, J.; Juwet, M. Design and Calibration of an Instrumented Seat Post to Measure Sitting Loads While Cycling. Sensors 2020, 20, 1384.

25. D'hondt, J.; Dieltiens, S.; Juwet, M. Design and Calibration of a 6-component Balance in a Bicycle Steer. In Proceedings of the 18th International Conference on Experimental Mechanics (ICEM18), Brussels, Belgium, 1 July 2018. 
26. Dieltiens, S.; Claeys, K.; D’hondt, J.; Devroey, H.; Juwet, M.; Demeester, E. Validation of a Bar Linkage Model for Joint Angle Estimation during Cycling. Appl. Sci. 2020, 10, 5104. [CrossRef]

27. Tikkanen, O. Physiological Loading during Normal Daily Life and Excercise Assessed with Electromyography; University of Jyvaskyla: Jyvaskyla, Finland, 2014.

28. Pesola, O. Reduced Muscle Inactivity, Sedentary Time and Cardio-Metabolic Benefits; University of Jyvaskyla: Jyvaskyla, Finland, 2016.

29. Colyer, S.I.; McGuigan, P.M. Textile electrodes embedded in clothing: A practical alternative to traditional surface electromyography when assesing muscle excitation during functional movements. J. Sport Sci. Med. 2018, 17, 101-109.

30. Holmes, J.C.; Pruitt, A.L.; Whalen, N.J. Lower Extremity overuse in bicycling. Clin. Sports Med. 1994, 13, 187-203. [CrossRef]

31. Burke, E.R. Perfect positioning. In Serious Cycling, Champaign (IL); Human Kinetics: Champaign, IL, USA, 2002 ; pp. $235-245$.

32. Burden, A. How should we normalize electromyograms obtained from healthy participants? What we have learned from over 25 years of research. J. Electromyogr. Kinesiol. 2010, 20, 1023-1035. [CrossRef]

33. Yanamashetti, G.; Murthy, H.S. Application of Global Regression method for Calibration of Wind Tunnel. In Proceedings of the Symposium on Applied Aerodynamics and Design of Aerospace Vehicles, Bangalore, India, 16-18 November 2011.

34. Carpes, F.P.; Mota, C.B.; Faria, I.E. On the bilateral asymmetry during running and cycling-A review considering leg preference. Phys. Ther. Sport 2010, 11, 136-142. [CrossRef] [PubMed]

35. Carpes, F.P.; Rossato, M.; Faria, I.E.; Mota, C.B. Bilateral pedaling asymmetry during a simulated 40-km cycling time-trial. J. Sports Med. Phys. Fit. 2008, 47, 51-57. [CrossRef]

36. Costes, A.; Turpin, N.A.; Villeger, D.; Moretto, P.; Watier, B. A reduction of the saddle vertical force triggers the sit-stand transition in cycling. J. Biomech. 2015, 48, 2998-3003. [CrossRef]

37. Dorel, S.; Couterier, A.; Hug, F. Intra-session repeatability of lower limb muscles activation pattern during pedaling. J. Electromyogr. Kinesiol. 2008, 18, 857-865. [CrossRef] [PubMed]

38. Richmond, D.R. Handlebar problems in bicycling. Clin. Sports Med. 1994, 13, 165-173. [CrossRef]

39. Schwarzer, U.; Sommer, F.; Klotz, T.; Cremer, C.; Engelmann, U. Cycling and penile pressure: The type of saddle matters. Eur. Urol. 2002, 41, 139-143. [CrossRef]

40. Callaghan, M.J.; Phil, M. Lower body problems and injury in cycling. J. Bodyw. Mov. Ther. 2005, 9, 226-236. [CrossRef] 